\title{
Hyperchaotic Fractional-Order Systems and Their Applications
}

\author{
Ahmed Elsaid, ${ }^{1}$ Delfim F. M. Torres, ${ }^{2}$ Sachin Bhalekar, ${ }^{3}$ \\ Abdelalim Elsadany, ${ }^{4}$ and Amr Elsonbaty ${ }^{1}$ \\ ${ }^{1}$ Mathematics \& Engineering Physics Department, Faculty of Engineering, Mansoura University, P.O. 35516, Mansoura, Egypt \\ ${ }^{2}$ Department of Mathematics and CIDMA, University of Aveiro, 3810-193 Aveiro, Portugal \\ ${ }^{3}$ Department of Mathematics, Shivaji University, Kolhapur 416004, India \\ ${ }^{4}$ Department of Basic Science, Faculty of Computers and Informatics, Suez Canal University, Ismailia 41522, Egypt \\ Correspondence should be addressed to Ahmed Elsaid; a_elsaid@mans.edu.eg
}

Received 12 November 2017; Accepted 12 November 2017; Published 7 December 2017

Copyright (c) 2017 Ahmed Elsaid et al. This is an open access article distributed under the Creative Commons Attribution License, which permits unrestricted use, distribution, and reproduction in any medium, provided the original work is properly cited.

Research about fractional-order hyperchaotic systems gains a lot of interest from both theoretical and applied point of view. Some fractional-order hyperchaotic systems have been investigated, such as the fractional-order hyperchaotic Rossler system and the fractional-order hyperchaotic Chen system. Recent publications in this area include nonlinear circuits, secure communication, laser applications, spread spectrum communication, communication in star coupled network, video encryption communication, color image encryption algorithm, and applications of different types of synchronization.

We are pleased to announce the publication of this special issue focusing on novel topics in hyperchaotic fractional-order systems and their applications. The main objective of this special issue is to provide an opportunity to study the new developments related to novel chaotic systems, synchronization schemes, bifurcations, and control in hyperchaotic fractional-order systems along with their applications. Among the articles that were submitted for review, our editorial team has selected seven articles for publication. These articles cover the topics of adaptive fuzzy synchronization, image encryption algorithm, dynamical analysis of a novel hyperchaotic system, eigenvalue problems, BAM neural networks with distributed delays and impulses, complex synchronization scheme between integer-order and fractional-order chaotic systems with different dimensions, and fractional-order FPGA implementation. We are confident that this special issue advances the understanding and research of hyperchaotic fractional-order systems and their applications.

\section{Acknowledgments}

We would also like to thank all the reviewers who have participated in the review process of the articles submitted to this special issue.

Ahmed Elsaid

Delfim F. M. Torres

Sachin Bhalekar

Abdelalim Elsadany

Amr Elsonbaty 


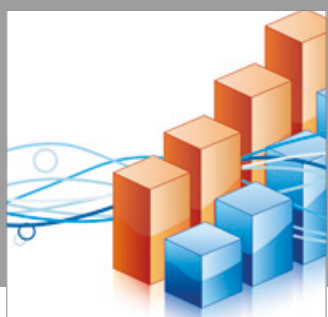

Advances in

Operations Research

vatersals

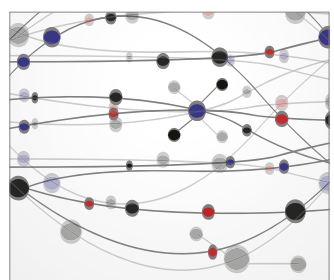

\section{The Scientific} World Journal
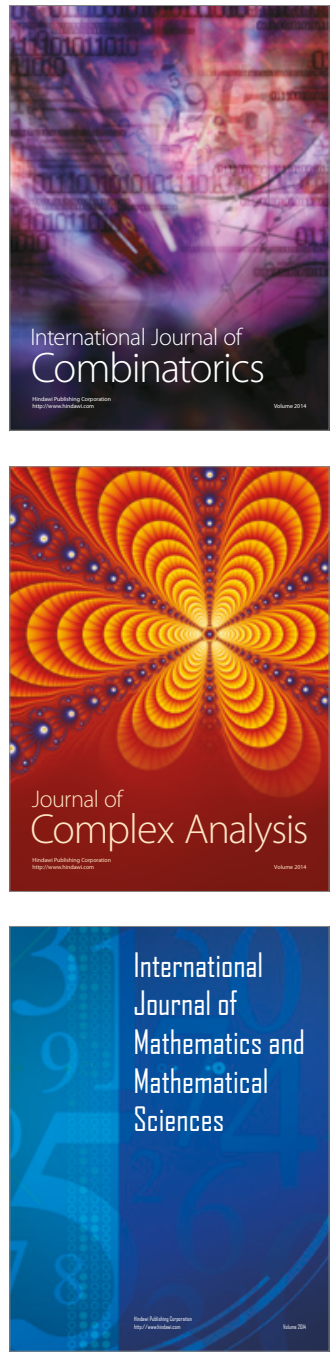
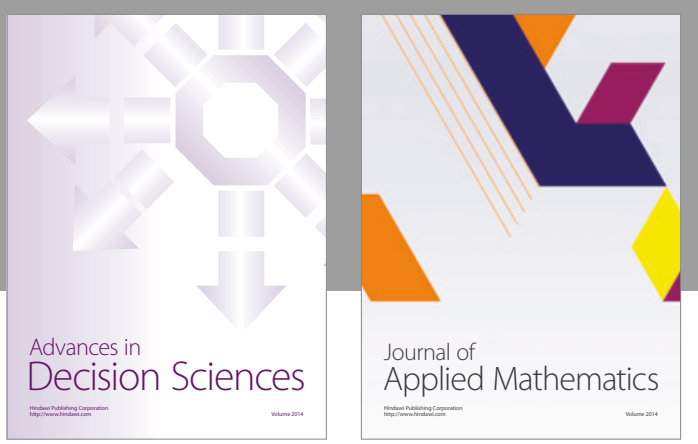

Algebra

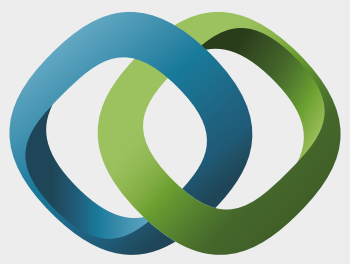

\section{Hindawi}

Submit your manuscripts at

https://www.hindawi.com
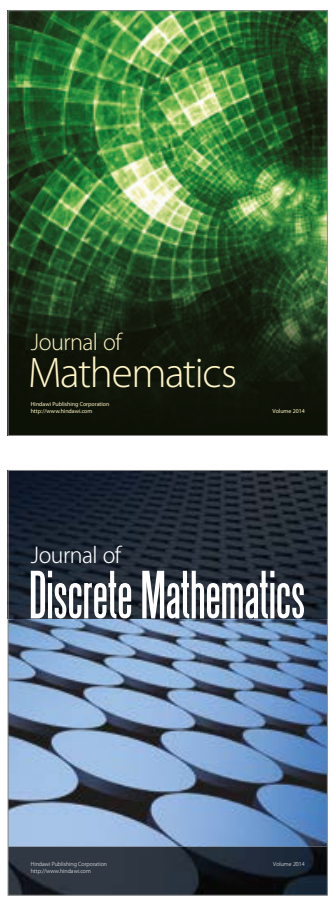

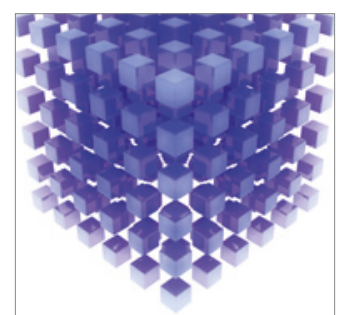

Mathematical Problems in Engineering
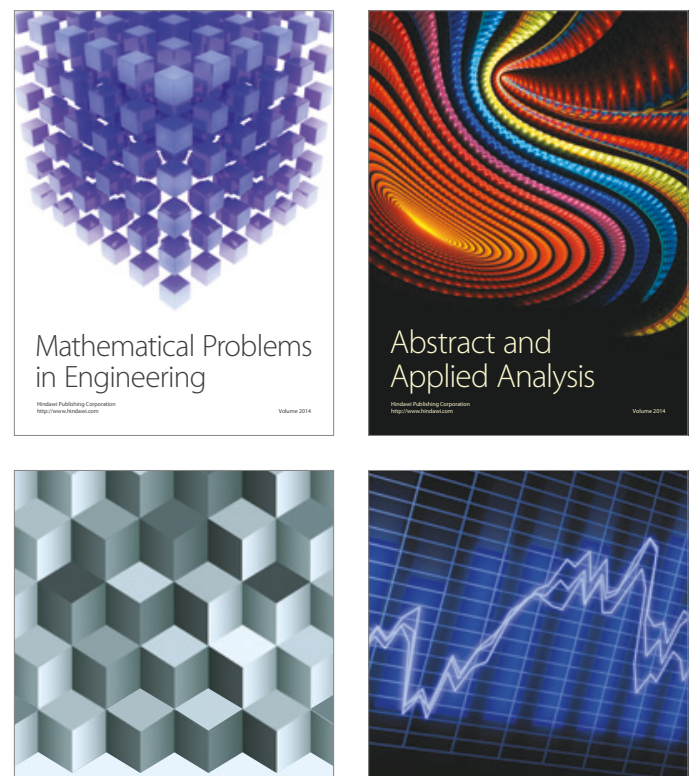

Journal of

Function Spaces

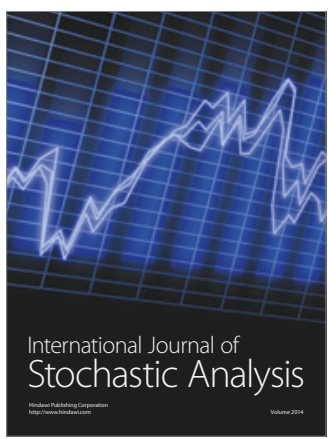

Probability and Statistics
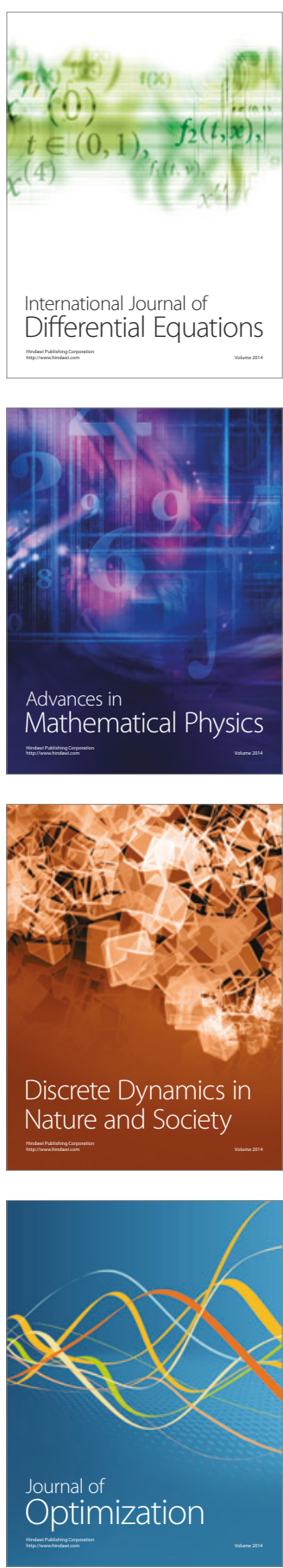